\title{
Integrating Static Checking and Interactive Verification: Supporting Multiple Theories and Provers in Verification
}

\author{
Joseph R. Kiniry ${ }^{1}$, Patrice Chalin ${ }^{2}$, and Clément Hurlin ${ }^{3}$ \\ ${ }^{1}$ Systems Research Group \\ School of Computer Science and Informatics \\ UCD Dublin \\ Belfield, Dublin 4, Ireland \\ ${ }^{2}$ Dependable Software Research Group \\ Department of Computer Science and Software Engineering \\ Concordia University \\ Montreal, Quebec, H3G 1M8, Canada \\ ${ }^{3}$ Université Henri Poincaré, Nancy 1 \\ BP 60120, Nancy Cedex, France \\ with contributions from \\ Cees-Bart Breunesse, Julien Charles, David Cok, Bart Jacobs, \\ Erik Poll, Silvio Ranise, Aleksy Schubert, and Cesare Tinelli
}

\begin{abstract}
Automatic verification by means of extended static checking (ESC) has seen some success in industry and academia due to its lightweight and easyto-use nature. Unfortunately, ESC comes at a cost: a host of logical and practical completeness and soundness issues. Interactive verification technology, on the other hand, is usually complete and sound, but requires a large amount of mathematical and practical expertise. Most programmers can be expected to use automatic, but not interactive, verification. The focus of this proposal is to integrate these two approaches into a single theoretical and practical framework, leveraging the benefits of each approach.
\end{abstract}

\section{Introduction}

Endemic in society today are problems related to the lack of software quality which, as a result, is costing governments, businesses, and nations billions of dollars annually [18]. Correctness and security issues are also directly related to some of the most important concerns of the day such as those of national security and technology-based voting. Additionally, driven by governmental regulations and market demands, businesses are now slowly beginning to assume liability for the faults exhibited by the software systems they offer to their customers. This is particularly true in safety and security critical domains.

While a variety of software engineering practices have been developed to help increase software quality (e.g., testing practices, system design, modern processes, robust operating systems and programming languages), it is widely acknowledged that a promising way to achieve highly reliable software in critical domains is to couple 
these practices with applied formal techniques supported by powerful modern tools and technologies like those discussed in this paper.

\subsection{Program Verification}

Applied formal methods has turned a corner over the past few years. Various groups in the semantics, specification, and verification communities now have sufficiently developed mathematical and tool infrastructures that automatic and interactive verification of software components that are written in modern programming languages like Java has become a reality. Automatic verification by means of Extended Static Checking (ESC) has seen some success in industry and academia due to its lightweight and easy-to-use nature. Unfortunately, ESC comes at a cost: a host of logical and practical completeness and soundness issues. Interactive verification technology, on the other hand, is usually complete and sound, but requires a large amount of mathematical and practical expertise. Typical programmers can be expected to use automatic, but not interactive, verification.

In this paper we discuss work which is being undertaken to:

- integrate the ESC and interactive verification approaches into a single theoretical framework, thus creating a unified semantic foundation, and

- directly realize this theoretical framework in a modern software development environment (IDE) as an Open Source initiative.

Specifically, our current work is focused on the integration of the verification technologies behind two successful tools, namely ESC/Java2 [5] and the LOOP program verifier [12] (both will be described shortly). The proposed integrated environment will perform as much automated verification as possible, falling back on interactive verification only when necessary. Additionally, in those situations where developers wish to delay the completion of the interactive proofs, the tool will insert run-time assertion checking code.

\section{Two Key Java Verification Tools}

Next, we discuss two complementary verification tools for Java upon which we base this work. These two tools are complementary because one is an automatic checker and the other is an interactive one.

\subsection{Extended Static Checking: ESC/Java2}

One of the most successful automatic verification tools for Java has been ESC/Java, an extended static checker originally developed at DEC SRC [7]. The next-generation release, called "ESC/Java2", is now available as an Open Source project that is supported by academic and industrial researchers [13]. David Cok and the first author are the ESC/Java2 project administrators and have been the main contributors (until recently). ESC/Java2 is currently used as a research foundation by over a half dozen research groups and as an instructional tool in nearly two dozen software-centric courses around the world. 
ESC/Java2 reasons about Java programs that are specified with annotations written in the Java Modeling Language (JML) [2]14]. ESC/Java2 automatically converts JMLannotated Java code into verification conditions that are automatically discharged by an embedded theorem prover-currently, Simplify [6]. Problems in the specifications, programs, or the checking itself are indicated to the user by means of error messages. As ESC/Java2's performance and mode of interaction are comparable to an ordinary compiler, it is quite usable by industry developers as well as computer science and software engineering students.

\subsection{Interactive Verification: The LOOP Tool}

The LOOP tool, developed by the SoS Group at Radboud University Nijmegen under the supervision of Prof. Bart Jacobs, is an interactive verification tool for JavaCard [3]. The LOOP tool is one of the most complete verifiers with respect to the subset of Java that it covers. LOOP compiles JML-annotated Java programs into proof obligations expressed as theories for the PVS theorem prover. By making use of PVS to interactively discharge the proof obligations, one is able to prove a program correct with respect to its JML specification.

The base Java/JML semantics of the LOOP tool essentially consists of a parameterized theory. The theory parameters are for the (sub-)theory to be used to reason about integral types. Early in the LOOP Project, Java's integral types were modeled by the mathematical integers. Later, support was added for bounded integers (with the familiar modulo arithmetic) and a bitvector representation (which facilitates reasoning about bit-wise operations - something that is common in JavaCard applications). When reasoning about Java programs, one has a choice of program logics including Hoare logics and two weakest precondition calculi. Recently, Breunesse has merged these into a single, unified theory in which different representations can be used simultaneously [1].

As these two tools represent some of the best-of-breed of applied formal methods in the Java domain, integrating their foundations and approaches has merit. To accomplish this goal, there are several theoretical and practical challenges to be faced.

\section{Integration: Observations and Challenges}

There is no single canonical semantics of Java. The canonical informal semantics for Java is embodied in the Java Language Specification [9]. Various groups have formalized portions of this text and built complementary tools, e.g., the

- Everest Group at INRIA Sophia-Antipolis,

- SoS Group at the Radboud University Nijmegen,

- Logical Group at INRIA Futurs/Université Paris-Sud,

- SAnToS Laboratory at Kansas State University,

- KeY group, composed of researchers from the Chalmers University of Technology, the University of Koblenz, and the University of Karlsruhe,

- Software Component Technology Group at ETH Zürich, and

- now disbanded Extended Static Checking Group at Hewlett-Packard/Compaq/Digital Systems Research Center. 
In all of these cases the formalizations are incomplete, either in scope or in accuracy. Also, very little is understood about how the various semantics relate to each other.

There is no single, core, canonical semantics of JML. While there are several partial informal and formal semantics for JML, there is no single, core semantics. Furthermore, the informal semantics of JML is much more transient and imprecise than that of Java, so the problems mentioned above for Java are compounded for JML. This state of affairs leads to subtle inconsistencies between the interpretation of specifications by the tools that support JML. Because of this inconsistency, relating the semantics to each other is extremely difficult. Additionally, explaining, extending, and reasoning about these artifacts (e.g., the calculi of ESC/Java2) is very difficult.

Little work has been done on meta-logical reasoning about object logics. By metalogical reasoning we mean reasoning about, rather than within, the semantics of program and specification languages. Formal meta-mathematical proofs are rare. It is not known, for example, if ESC/Java2's object logic is sound. This is a critical issue.

\section{An Integrated Verification Environment}

In collaboration with others, our research groups have begun work on an integrated verification environment (IVE) and its necessary theoretical foundations. In doing so we have started to address the problems identified in the previous section.

We are (concurrently) working on the achievement of the following initial milestones:

- elaboration of a semantics for a "core" JML,

- extracting, analysing, and extending ESC/Java2's logic and calculi, and

- redesigning ESC/Java2's proof infrastructure as well as backend interfaces and adaptors with the main objective of allowing it to support new provers.

\subsection{Semantics for JML}

Semantics have been developed for JML within different logics, nearly all of which have been embedded in the various tools developed by the groups enumerated in Section 3 A few of these tools are publicly available, but most were never used outside the group that originally developed them.

To resolve ambiguities, disagreements, and lack of detailed formal documentation within the JML community, a single, open semantics of a "core" of JML (recently named JML Level 1) needs to be written. Chalin and Kiniry are currently outlining a proposed core and have begun formalizing its definition. The outcome of this effort is also a major goal of the MOBIUS project [15].

This semantics will be written in a well-understood formalism, e.g., within a modern extension to Hoare logic, a denotational semantics, and/or in an operational semantics. In our initial work we have decided to express our base, canonical semantics in PVS and Isabelle. Realizing the object logic within higher-order provers will help us characterize and compare semantics.

It is expected that multiple formalizations of the object logic will be created due to practical and theoretical reasons. E.g., most research groups have developed expertise 
in only one prover, and furthermore, the community can benefit from experimentation with the varying capabilities of each of these provers.

\subsection{Evolving ESC/Java2's Logic and Calculi}

As inherited from its predecessor, SRC's ESC/Java, ESC/Java2 makes use of an unsorted object logic and two calculi (a weakest precondition calculus and a strongest postcondition calculus). The unsorted object logic consists of approximately 80 axioms written in the language understood (only) by the Simplify prover. These axioms are highly tuned to the quirks and capabilities of Simplify. Initial logical extensions in ESC/Java2 saw the logic augmented with approximately another 20 axioms.

A transcription of this Simplify-based unsorted object logic has been written in PVS. We refer to this formalization of the logic as $\mathrm{EJ}_{0}$. Two other logics, $\mathrm{EJ}_{1}$ and $\mathrm{EJ}_{2}$, have also been written; $\mathrm{EJ}_{1}$ is merely a sorted version of $\mathrm{EJ}_{0}$ whereas $\mathrm{EJ}_{2}$, also a sorted logic, was written from scratch with the purpose of better representing the abstractions needed by ESC/Java2 to reason about JML annotated Java programs. Soundness proofs as well as results on the (semi-)equivalence of the $\mathrm{EJ}_{i}$ logics are underway.

We will also be "extracting" the weakest precondition and a strongest postcondition calculi of ESC/Java2, as well as at least one of the weakest precondition calculi used with the LOOP verification system. This will most likely be done in a higher-order logic or a term rewriting framework. The rewriting speed of special purpose environments like Maude [4] may be of benefit as the tool and verification efforts scale to larger problems.

\subsection{Supporting Multiple Provers}

As we progress in our work on the definition and proofs of soundness and completeness of the $\mathrm{EJ}_{i}$ logics, we are also progressing in our work on extending and adapting ESC/Java2 to support multiple provers. By developing a generic prover interface along with suitable adaptors, we plan on experimenting with next-generation first-order provers, and a few higher-order provers.

We anticipate the possibility of supporting the use of multiple provers, simultaneously or independently. Which prover to use might be determined automatically by ESC/Java2 based on the context of the verification and the capabilities of the provers. For example, while Simplify is a very fast predicate solver, it does not support a complete or sound (fragment of) arithmetic, thus in verification contexts where arithmetic is used, the tool should automatically avoid using Simplify.

We have chosen Sammy and haRVey as the initial provers for experimentation [8]16]. This choice was made due to our research relationship with the authors of these two tools as well as the authors' high-profile position within the SMT-LIB community [19].

As a necessary precursor to being able to support multiple provers, we are required to translate our object logic, whose current canonical representation is in PVS, into an appropriate formalism understood by each of the provers. Encoding of the ESC/Java2 object logic for these provers is being accomplished primarily by their respective research teams.

We will also be experimenting with the use of higher-order provers as backends for ESC/Java2. Our initially targeted provers are PVS, Isabelle, and Coq. Aside from the 
authors, Julien Charles at INRIA is working on a Coq realization of the object logic and Cesare Tinelli is contributing to the PVS realization.

\section{Conclusion}

One of the advantages of our project is that we have a working toolset today that supports Java and JML. These tools are actively being used by researchers and a few industry practitioners. Our goal is to help evolve these tools into their next-generation counterparts and, all the while, make sure that we take our own medicine. Thus, for example, writing JML specifications for the Java modules of our toolsets has been and is routinely done. We are also applying our tools to themselves, thus providing non-trivial case studies demonstrating the practical utility of the tools.

ESC/Java2 and LOOP have been applied to other case studies in the areas of Internet voting [11], JavaCard applications [10], and web-based enterprise applications [17], for example. Some of these case studies are already part of our GForge [20]. We will be routinely re-executing these case studies as the tools evolve so as to validate the tools and ensure that their effectiveness is, in fact, improving.

\section{Acknowledgments}

This proposal is based upon the work of many people. Our collaborators are gratefully acknowledged on the first page as well as in the various sections of the proposal. We thank the anonymous referees for their helpful comments. This work is being supported by the Ireland Canada University Foundation as well by the European Project Mobius within the frame of IST 6th Framework and national grants from the Science Foundation Ireland and Enterprise Ireland. This paper reflects only the authors' views and the Community is not liable for any use that may be made of the information contained therein.

\section{References}

1. Breunesse, C.-B.: On JML: Topics in Tool-assisted Verification of Java Programs. PhD thesis, Radboud University Nijmegen (2006)

2. Burdy, L., Cheon, Y., Cok, D., Ernst, M., Kiniry, J., Leavens, G.T., Leino, K.M., Poll, E.: An overview of JML tools and applications. International Journal on Software Tools for Technology Transfer (STTT) 7(3), 212-232 (2005)

3. Chen, Z.: Java Card Technology for Smart Cards: Architecture and Programmer's Guide (2000)

4. Clavel, M., Durán, F., Eker, S., Meseguer, J., Stehr, M.-O.: Maude as a formal meta-tool. In: Proceedings of the World Congress on Formal Methods in the Development of Computing Systems (1999)

5. Cok, D.R., Kiniry, J.R.: ESC/Java2: Uniting ESC/Java and JML. In: Barthe, G., Burdy, L., Huisman, M., Lanet, J.-L., Muntean, T. (eds.) CASSIS 2004. LNCS, vol. 3362, pp. 108-128. Springer, Heidelberg (2005)

6. Detlefs, D., Nelson, G., Saxe, J.B.: Simplify: a theorem prover for program checking. J. ACM 52(3), 365-473 (2005) 
7. Flanagan, C., Leino, K.R.M., Lillibridge, M., Nelson, G., Saxe, J.B., Stata, R.: Extended static checking for Java. In: ACM SIGPLAN 2002 Conference on Programming Language Design and Implementation (PLDI 2002), pp. 234-245 (2002)

8. Ganzinger, H., Hagen, G., Nieuwenhuis, R., Oliveras, A., Tinelli, C.: DPLL(T): Fast decision procedures. In: Alur, R., Peled, D.A. (eds.) CAV 2004. LNCS, vol. 3114, pp. 175-188. Springer, Heidelberg (2004)

9. Gosling, J., Joy, B., Steele, G.: The Java Language Specification, 1st edn. (August 1996)

10. Jacobs, B.: JavaCard program verification. In: Boulton, R., Jackson, P. (eds.) Theorem Proving in Higher Order Logics TPHOL 2001, vol. 2151, pp. 1-3 (2001)

11. Jacobs, B.: Counting votes with formal methods. In: Rattray, C., Maharaj, S., Shankland, C. (eds.) AMAST 2004. LNCS, vol. 3116, pp. 21-22. Springer, Heidelberg (2004)

12. Jacobs, B., Poll, E.: Java program verification at Nijmegen: Developments and perspective. In: Futatsugi, K., Mizoguchi, F., Yonezaki, N. (eds.) ISSS 2003. LNCS, vol. 3233, pp. 134 153. Springer, Heidelberg (2004)

13. Kiniry, J.R., Cok, D.R.: ESC/Java2: Uniting ESC/Java and JML: Progress and issues in building and using ESC/Java2 and a report on a case study involving the use of ESC/Java2 to verify portions of an Internet voting tally system. In: Barthe, G., Burdy, L., Huisman, M., Lanet, J.-L., Muntean, T. (eds.) CASSIS 2004. LNCS, vol. 3362, pp. 108-128. Springer, Heidelberg (2005)

14. Leavens, G.T., Poll, E., Clifton, C., Cheon, Y., Ruby, C., Cok, D., Kiniry, J.: JML Reference Manual. Department of Computer Science, Iowa State University, 226 Atanasoff Hall, draft revision 1.94 edition (2004)

15. The MOBIUS project.http://mobius.inria.fr/

16. Ranise, S., Deharbe, D.: Light-weight theorem proving for debugging and verifying units of code. In: International Conference on Software Engineering and Formal Methods SEFM 2003, Canberra, Australia (September 2003)

17. Rioux, F., Chalin, P.: Improving the quality of web-based enterprise applications with extended static checking: A case study. Electronic Notes in Theoretical Computer Science 157(2), 119-132 (2006)

18. RTI: Health, Social, and Economics Research, Research Triangle Park, NC. The economic impacts of inadequate infrastructure for software testing. Technical Report Planning Report 02-3, NIST (May 2002)

19. SMT-LIB: The satisfiability modulo theories library. http://goedel.cs.uiowa.edu/smtlib/

20. The Systems Research Group GForge.http://sort.ucd.ie/

\section{A Discussion on Joseph Kiniry's Presentation}

[Demo by Joseph Kiniry, requested by Eric Hehner.]

\section{Bertrand Meyer}

Don't you want your students to use some kind of specification whatsoever?

\section{Joseph Kiniry}

Oh, very much so! I teach in a style where we do Design by Contract, you know all that, Bertrand. So, we do all that. But oftentimes, you are dealing with code that has 
no annotations to begin with, and it is a bootstrapping problem. The students want to know what utility they get out of tools when there are no annotations at all. And that is where you start doing the game that Rustan was playing, where you have code that has no annotations and use the tool to give you feedback to make changes, and basically do what I call "contracting the design", which is unfortunately the way most people work with the tools, even when we keep educating them about the "dual". You have to be able to give them a tool where they actually can start out from nothing, in a sense. And that is what this tool lets you do, because there is a lot of implicit annotations inside here besides all these fancy ones with data refinement and data group support and heavyweight specs and all these kinds of things. So, in a sense, the only reason we do it this way is because we can, and it helps people to bootstrap and to understand, how to write specifications without forcing them to do design by contract initially, which we teach in a different course. So, this is how we gently introduce them to this sort of tool use. So, pedagogically, it might not be the right way, but it is a nice way to slip it in. I call it "Secret Ninja Formal Methods".

\section{Bertrand Meyer [after some silence]}

If no one else is speaking, I'll just add to what Joseph just said, just to fill the silence, that here at ETH contracts come in week two, lecture three actually, of our first-semester "Introduction to programming" course. 